\title{
PERFIL DA PRODUÇÃO DE UMA PUBLICAÇÃO CIENTÍFICA: REVISTA LATINO- AMERICANA DE ENFERMAGEM
}

\author{
THE PROFILE OF A SCIENTIFIC JOURNAL: REVISTA LATINO-AMERICANA DE \\ ENFERMAGEM (LATIN AMERICAN JOURNAL OF NURSING) \\ PERFIL DE LA PRODUCCIÓN DE UNA PUBLICACIÓN CIENTÍFICA: REVISTA \\ LATINOAMERICANA DE ENFERMERÍA
}

Maria Helena Palucci Marziale ${ }^{1}$ Isabel Amélia Costa Mendes ${ }^{2}$

\begin{abstract}
RESUMO: O artigo apresenta a produção divulgada pela Revista Latino - americana de Enfermagem no período de 1993 a setembro de 2001, o destino dos manuscritos submetidos à publicação e a descrição dos principais problemas detectados pelos editores e consultores referentes à avaliação dos manuscritos. Apesar dos problemas detectados a maioria dos manuscritos elaborados pelos enfermeiros pesquisadores foi considerada de boa qualidade, contribuido de forma significativa para o desenvolvimento do conhecimento da Enfermagem. O problema apontado tem por finalidade alertar os autores sobre alguns aspectos a serem observados antes do encaminhamento dos manuscritos a um periódico científico para publicação.

PALAVRAS-CHAVE: produção cientifica, divulgação, editoração
\end{abstract}

ABSTRACT: This article presents the production disseminated by Revista Latino-Americana de Enfermagem from 1993 to September 2001, the destination of the manuscripts submitted for publication and the description of the major problems detected by the editors and reviewers concerning manuscript evaluation. In spite of the problems detected, most of the manuscripts elaborated by nurse researchers were considered to be good quality and to have significantly contributed to nursing development. The problem that has been pointed out aims at warning authors with regard to some aspects that must be observed before submitting manuscripts to a journal for publication.

KEYWORDS: scientific production, dissemination, editing

RESUMEN: El artículo presenta la producción divulgada por la Revista Latinoamericana de Enfermeria en el periodo entre 1993 y septiembre de 2001, el destino de los manuscritos sometidos a publicación y la descripción de los principales problemas detectados por los editores y consultores referientes a la evaluación de los manuscritos. Además de los problemas detectados, la mayoria de los manuscritos elaborados por los enfermeros investigadores fue considerada de buena calidad, contribuyendo de forma significativa para el desarrollo del conocimiento de la Enfermeria. El problema apuntado tiene por finalidad alertar a los autores sobre algunos aspectos a seren observados antes del encaminamiento de los manuscritos a un periódico científico para publicación.

PALABRAS CLAVE: producción científica, publicación, editoración

Recebido em 12/12/2001

Aprovado em 12/02/2002

\footnotetext{
${ }^{1}$ Presidente da Comissão de Editoração da Revista Latino-Americana de Enfermagem. Professor Livre-Docente da Escola de Enfermagem de Ribeirão Preto /USP. Centro Colaborador da OMS para o desenvolvimento de pesquisa em enfermagem.

2 Vice-Presidente da Comissão de Editoração da Revista Latino-americana de Enfermagem. Professor Titular da Escola de Enfermagem de Ribeirão Preto /USP. Centro Colaborador da OMS para o desenvolvimento de pesquisa em enfermagem
} 
INTRODUÇÃO

A Revista Latino-americana de Enfermagem é órgão oficial da Escola de Enfermagem de Ribeirão Preto/USP - Centro Colaborador da Organização Mundial da Saúde para o Desenvolvimento da Pesquisa em Enfermagem. Sua missão está voltada à publicação de artigos científicos relacionados à fundamentação das ações de enfermagem através da pesquisa científica visando a legitimação da profissão. O periódico é editado desde de 1993, publica artigos escritos em Português, Espanhol e Inglês, com periodicidade bimestral, distribuída a assinantes dos Estados Unidos, Europa e vários paises da América Latina. Afiliada à Associação Brasileira de Editores Cientificos - ABEC, está indexada em três bases de dados internacionais International Index e Medline, Cumulative Index to Nursing \& Allied Health to Nursing - CINAHL e Literatura Latino-americana em Ciências da Saúde - LILACS e na base nacional BDEnf - Base de Dados em Enfermagem.

O periódico é gerenciado por um Conselho Diretor e pela Comissão de Editoração, a qual é responsável pela qualidade da publicação. Os artigos recebidos pela secretaria da revista são avaliados quanto ao cumprimento das normas de publicação: quando é observado o descumprimento das normas, o manuscrito é devolvido ao autor para providências quanto à adequação; quando adequados e de interesse para a revista, são protocolados e encaminhados pelos editores a dois consultores para emissão de parecer.

A revista conta com 39 consultores ( $54 \%$ pertencentes a instituições nacionais e $46 \%$ a internacionais) e 100 consultores "Ad-Hoc", os quais analisam $\mathrm{o}$ artigo com base em um instrumento elaborado pela Comissão de Editoração. Os consultores são pesquisadores de reconhecido saber com titulação minima de Doutor.

Após a emissão dos pareceres, os mesmos são analisados pelos editores, quando da necessidade de reformulação são enviados aos autores para ajustes. Os artigos podem ser aceitos para publicação, recusados ou reformulados; neste caso, podem ser enviados aos autores até três vezes visando sua adequação. Destacamos que este processo tem atrasado o periodo de publicação dos artigos e necessita ser revisto.

Os artigos aceitos para publicação pelos consultores são aprovados e categorizados pela comissão de editoração (artigo original, revisão, atualização, notas e informações, página do estudante e resenhas) e posteriormente são encaminhados à bibliotecária para ajustes nas referências bibliográficas e a tradutores da lingua inglesa e espanhola para revisão do resumo e de todo o texto quando não escrito em Português.
Diante da missão de publicação de artigos científicos que fundamentem a Enfermagem enquanto profissão e do desenvolvimento de conhecimento na área, a comissão de editoração tem a constante preocupação de divulgar aos leitores artigos de qualidade, tarefa esta efetuada com a colaboração dos consultores.

$\mathrm{Na}$ etapa final de produção da publicação o número é organizado com cerca de15 artigos, sendo utilizado como critério de inclusão a data do recebimento do artigo e a data de aprovação para publicação. Cada número pode ser composto por até $50 \%$ de artigos de autores internos à Unidade e 50\% externos, a fim de evitar a entropia e cumprir a missão do periódico que é a disseminação do conhecimento de enfermagem produzido no Brasil e na AméricaLatina. Antes de ser enviado para impressão o material é integralmente revisado.

A elaboração da revista, objeto desta análise, sofre modificações que fazem parte da evolução gradual, comum aos periódicos científicos. Um exemplo das modificações introduzidas recentemente foi a adoção de critérios de registro de dados de recebimento do artigo pela revista, acompanhado pela data de sua aprovação para publicação. Este é um tipo de indicador de garantia de originalidade e de crédito de procedência. Considerando o hiato temporal entre a submissão do artigo e sua publicação por um periódico, é preciso que se dê ao autor o crédito de prioridade. Assim fica esclarecida a questão sobre quem fez determinada abordagem em primeiro lugar.

Os registros destas datas servem também como indicador do fluxo da revista e os autores (clientes em potencial) podem valer-se deste dado, dentre outros, para selecionar o periódico ao qual vai submeter seu manuscrito. Passou este a ser, também, um dos itens na avaliação de qualidade dos periódicos.

Como é possivel observar, a editoração do periódico possui uma longa etapa operacional e quando acrescida 'a aspectos organizacionais, estruturais e financeiros torna-se bastante trabalhosa e onerosa .

Durante o processo de editoração vários problemas são enfrentados, entre os quais destacamos os custos de postagem. A fim de minimizar os gastos dessa natureza estamos enviando parte do material através de via eletrônica durante o processo de avaliação por pares. Ainda no que se refere a custos, a Revista não é auto-sustentável com a verba advinda da taxa de assinaturas; o fomento recebido pelas agências financiadoras ( Fundação Kellog, Fundação de Amparo a Pesquisa do Estado de São Paulo-FAPESP e Conselho Nacional de Pesquisa-CNPq) sempre foi imprescindivel para a manutenção do periódico.

O atraso na emissão dos pareceres pelos 
consultores e na devolução dos artigos pelos autores tem contribuido de forma significativa para aumentar o prazo entre o cadastro do manuscrito e a publicação do artigo. Cabe destacar que a demanda dos manuscritos recebidos para análise é, também, fator interferente no prazo de espera. A partir de 2001 a periodicidade da Revista Latino-americana de Enfermagem passou a ser bimestral vislumbrando agilizar a divulgação dos artigos e a diminuição do prazo de espera.

Dentre as mudanças implementadas no ano de 2001 visando o alcance de melhor qualificação, as normas de publicação foram modificadas e a revista passou a adotar os Requisitos Uniformes para manuscritos apresentados a periódicos biomédicos "estilo Vancouver" (INTERNATIONAL COMMITTEE, 1997), cujas vantagens de sua adoção estão relacionadas à clareza da apresentação do texto, maior destaque ao posicionamento do autor frente à pesquisa apresentada, economia de espaço possibilitando a publicação de maior quantidade de artigos por número e/ou redução de custos e a possibilidade de ter o periódico indexado em outras bases de dados.

Todas as mudanças introduzidas na Revista Latino- Americana de Enfermagem estiveram relacionadas com a complexidade e crescimento da comunidade científica, com a necessidade de aperfeiçoar a comunicação e com o sistema de avaliação editorial.

Com a finalidade de levantar quantitativamente a produção científica divulgada pela Revista LatinoAmericana de Enfermagem desde o primeiro número editado e conhecer os principais problemas das pesquisas submetidas à apreciação, segundo opinião dos consultores realizamos este estudo cujos objetivos foram:

- Levantar a produção divulgada pela Revista Latino-Americana de Enfermagem e o destino dos manuscritos submetidos para divulgação;

- Identificar os principais problemas detectados pelos consultores nos manuscritos analisados para publicação.

\section{MÉTODOS}

Trata-se de um estudo exploratório, descritivo realizado através de duas fases de execução.

$1^{\text {a }}$ fase - Realização de levantamento documental através do Banco de Dados da Revista Latino-Americana de Enfermagem visando o levantamento do destino dos manuscritos recebidos para publicação.

$2^{\mathrm{a}}$ fase - Encaminhamento à população de consultores da Revista Latino-Americana de Enfermagem ( $n=139)$, através de correio eletrônico na primeira quinzena de setembro de 2001, a seguinte questão norteadora:

- Quais foram os principais problemas detectados nos artigos que the enviamos para emissão de parecer, principalmente no que se refere à qualidade dos artigos?

Ressaltamos que os consultores foram informados que os resultados da coleta de dados seria divulgado e que seria mantido sigilo quanto à identificação dos mesmos.

A amostra foi composta pelas respostas encaminhadas por $58(41,7 \%)$ consultores que atenderam livremente à solicitação feita.

Análise dos dados

Os dados coletados na primeira fase do estudo foram analisados através de cálculos percentuais simples e as respostas obtidas através da questão norteadora, foram agrupadas nas seguintes categorias: temática, objetivos, referencial teóricometodológico, análise e tratamento dos dados, resultados e discussão, conclusões, resumos, contribuição para o conhecimento em Enfermagem, redação e outros.

\section{RESULTADOS E DISCUSSÃO}

Inicialmente são apresentados os dados relativos a produção científica publicada pelo periódico desde 1993. A tabela 1 ilustra os resultados obtidos no levantamento documental realizado.

No periodo compreendido entre janeiro de 1993 e setembro de 2001 foram recebidos para análise 788 manuscritos, sendo observado um aumento numérico na demanda a partir de 1998. Dos manuscritos recebidos para análise $50(6,3 \%)$ foram recusados e $111(14,1 \%)$ cancelados. Cabe ressaltar que o motivo geralmente detectado nos casos de cancelamentos estava relacionado às necessidades de reformulações visando a adequação dos manuscritos.

Dentre as justificativas emitidas pelos consultores indicativas de recusa das publicações destacamos a inobservância estrutural do manuscrito como trabalho científico, desacato às recomendações de reformulação, não alcance das propostas formuladas pelo estudo, inadequação do referencial teórico-metodológico, incoerência na análise e discussão dos resultados, não cumprimento dos requisitos éticos de pesquisas com seres humanos e a não contribuição para o desenvolvimento do conhecimento da Enfermagem.

Ainda, na tabela 1 podem ser observados os dados relativos aos manuscritos que estão em processo de publicação. Atualmente 51 manuscritos estão com os autores para providências quanto as reformulações sugeridas, 118 estão sendo analisados pelos conselheiros e 33 artigos estão aprovados aguardando publicação. 
TABELA 1 - Distribuição dos manuscritos recebidos para publicação na Revista Latino-Americana de enfermagem no período de 1993 a setembro de 2001, segundo número de manuscritos recusados, em tramitação, cancelados, artigos no prelo e artigos publicados

\begin{tabular}{|c|c|c|c|c|c|c|c|c|c|c|c|c|c|}
\hline \multirow[t]{3}{*}{ Período } & \multirow{3}{*}{$\begin{array}{c}\text { Manuscritos } \\
\text { Recebidos } \\
n\end{array}$} & \multirow{2}{*}{\multicolumn{2}{|c|}{$\begin{array}{c}\text { Manuscritos } \\
\text { Recusados }\end{array}$}} & \multirow{2}{*}{\multicolumn{2}{|c|}{$\begin{array}{r}\text { Manuscritos } \\
\text { Cancelados }\end{array}$}} & \multirow{2}{*}{\multicolumn{4}{|c|}{$\begin{array}{c}\text { Artigos em } \\
\text { tramitação } \\
\text { Autor Consultor }\end{array}$}} & \multicolumn{4}{|c|}{ Artigos } \\
\hline & & & & & & & & & & & prelo & Publ & cados \\
\hline & & $\mathrm{n}$ & $\%$ & $\mathrm{n}$ & $\%$ & $\mathrm{n}$ & f & $\mathrm{n}$ & f & $\mathrm{n}$ & $\%$ & $\mathrm{n}$ & $\%$ \\
\hline 1993 & 54 & 01 & 1,8 & 20 & 37,0 & 00 & - & 00 & - & 00 & - & 28 & 51,8 \\
\hline 1994 & 70 & 03 & 4,3 & 26 & 37,1 & 00 & - & 00 & - & 00 & - & 17 & 24,3 \\
\hline 1995 & 73 & 01 & 1,4 & 14 & 19,1 & 00 & - & 00 & - & 00 & - & 24 & 32,8 \\
\hline 1996 & 90 & 03 & 3,3 & 18 & 20,0 & 00 & - & 00 & - & 00 & - & 50 & 55,5 \\
\hline 1997 & 73 & 03 & 4,1 & 16 & 21,9 & 00 & - & 00 & - & 00 & - & 54 & 73,9 \\
\hline 1998 & 98 & 15 & 15,3 & 09 & 9,2 & 05 & 5,1 & 00 & - & 01 & 1,0 & 66 & 67,3 \\
\hline 1999 & 120 & 14 & 11,7 & 08 & 6,7 & 11 & 9,1 & 01 & 0,8 & 08 & 6,7 & 47 & 39,2 \\
\hline 2000 & 124 & 09 & 7,2 & 00 & - & 23 & 18,5 & 49 & 9,6 & 21 & 16,9 & 91 & 73,4 \\
\hline et/2001 & 86 & 01 & 1,2 & 00 & - & 12 & 14,0 & 68 & 79,0 & 03 & 3,5 & 59 & 68,6 \\
\hline Total & 788 & 50 & 6,3 & 111 & 14,1 & 51 & 6,5 & 118 & 15,0 & 33 & 4,2 & 436 & 55,3 \\
\hline
\end{tabular}

TABELA 2 - Distribuição dos artigos publicados na Revista Latino-Americana de Enfermagem no periodo de 1993 a setembro de 2001

\begin{tabular}{|c|c|c|c|}
\hline Ano & Número do Volume & Fascículos & Artigos Publicados \\
\hline \multirow{3}{*}{1993} & \multirow[t]{3}{*}{ ( } & 1 & 08 \\
\hline & & 2 & 11 \\
\hline & & Especial & 09 \\
\hline \multirow{2}{*}{1994} & \multirow{2}{*}{2} & l & 08 \\
\hline & & 2 & 09 \\
\hline \multirow{2}{*}{1995} & \multirow{2}{*}{3} & 1 & 12 \\
\hline & & 2 & 12 \\
\hline \multirow{4}{*}{1996} & \multirow{4}{*}{4} & 1 & 14 \\
\hline & & 2 & 11 \\
\hline & & 3 & 10 \\
\hline & & Especial & 15 \\
\hline \multirow{5}{*}{1997} & \multirow{5}{*}{5} & 1 & 11 \\
\hline & & 2 & 10 \\
\hline & & 3 & 11 \\
\hline & & 4 & 13 \\
\hline & & Especial & 09 \\
\hline \multirow{5}{*}{1998} & \multirow{5}{*}{6} & 1 & 12 \\
\hline & & 2 & 12 \\
\hline & & 3 & 13 \\
\hline & & 4 & 15 \\
\hline & & 5 & 14 \\
\hline \multirow{4}{*}{1999} & \multirow{4}{*}{7} & 1 & 11 \\
\hline & & 2 & 12 \\
\hline & & 3 & 11 \\
\hline & & 4 & 13 \\
\hline \multirow{6}{*}{2000} & \multirow{6}{*}{8} & 1 & 16 \\
\hline & & 2 & 15 \\
\hline & & 3 & 15 \\
\hline & & 4 & 13 \\
\hline & & 5 & 15 \\
\hline & & 6 & 17 \\
\hline \multirow{4}{*}{2001} & \multirow{4}{*}{9} & 1 & 16 \\
\hline & & 2 & 13 \\
\hline & & 3 & 16 \\
\hline & & 4 & 14 \\
\hline Total & 9 & 35 & 436 \\
\hline
\end{tabular}

Através da tabela 2 é apresentada a distribuição numérica dos artigos publicados (436) segundo o volume e número do fasciculo.

Desde a sua criação 436 artigos foram publicados através de 9 volumes e 35 fascículos, o que pode ser visualizado na tabela 2 . Foram editados 3 fasciculos especiais respectivamente, um em 1993, um em 1996 e um em 1997. A partir de então a Comissão de Editoração, após algumas ponderações, decidiu que não mais seria possivel edição de fascículos especiais. Através do quadro 1 (p. 35) são apresentadas as opiniões dos consultores em relação aos manuscritos avaliados por eles.

Apesar dos problemas mencionados os consultores consideram de boa qualidade a maioria dos manuscritos elaborados pelos enfermeiros pesquisadores, que apesar das dificuldades tem contribuido de forma significativa para o desenvolvimento do conhecimento da Enfermagem e que os problemas apontados tem por objetivo alertar os autores sobre alguns aspectos a serem observados antes do encaminhamento dos manuscritos a um periódico científico para publicação.

\section{CONSIDERAÇÕES FINAIS}

O presente texto foi preparado para ser compartilhado com editores de periódicos de enfermagem, em reunião, no $53^{\circ}$ Congresso Brasileiro de Enfermagem, promovido pela Associação Brasileira de Enfermagem -ABEn, dai o direcionamento das questões abordadas. Cumpre no entanto, ressaltar o valor da produção científica brasileira, publicada nesta e nas outras Revistas com que orgulhosamente contamos, e que com muito esforço mantemos. Nossa expectativa é a de que os enfermeiros continuem investindo no sentido de divulgar sua produção e que os problemas ou dificuldades, ora apontados, sejam 
encarados como possibilidade de crescimento e de enfrentamento de desafios e que, juntos, possamos contribuir para o progresso da Enfermagem enquanto ciência e enquanto arte.

QUADRO 1 - Opinião dos consultores da Revista Latino-americana de Enfermagem sobre a qualidade dos manuscritos submetidos para publicação segundo categorias de análise $(n=58)$

\begin{tabular}{|c|c|}
\hline Temática & $\begin{array}{ll}\text { - } & \text { falta de originalidade } \\
\text { - } & \text { tema desatualizado } \\
\text { - } & \text { não explicitação clara do problema de pesquisa } \\
\text { - } & \text { falhas na problematização e desarticulação das questões abordadas } \\
& \text { com a realidade } \\
\text { - } & \text { títulos inadequados }\end{array}$ \\
\hline Objetivos & $\begin{array}{ll}\text { - } & \text { definidos de forma vaga e desarticulada } \\
\text { - } & \text { falta clareza na sua elaboração } \\
\text { - } & \text { utilização incorreta do tempo verbal }\end{array}$ \\
\hline Referencialteórico - metodológico & 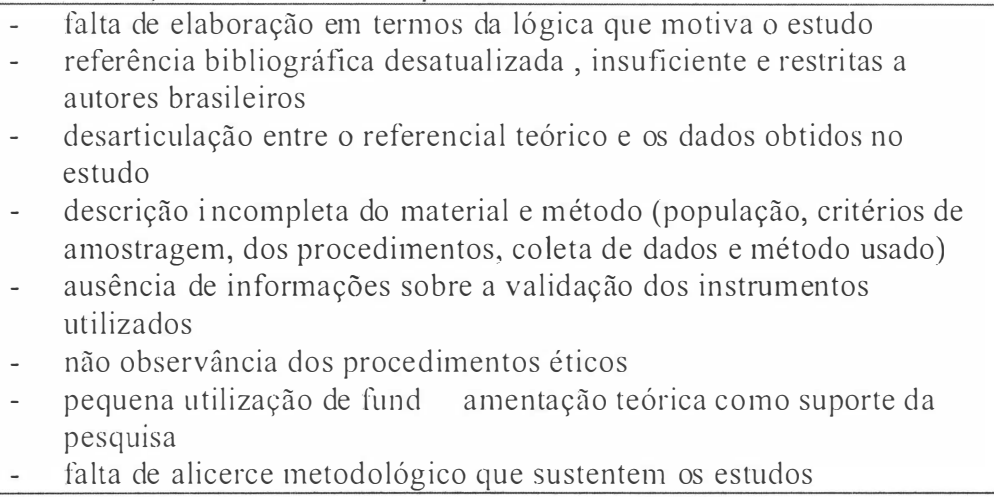 \\
\hline Análise dos dados & $\begin{array}{l}\text { - falta análise aprofundada dos dados e a utilização de recursos e } \\
\text { métodos disponiveis para análise (estatísticos etc..) } \\
\text { - } \quad \text { análise superficial, principalmente quando da abordagem qualitativa } \\
\text { - } \quad \text { ausência de referencial para análise }\end{array}$ \\
\hline Discussão dos resultados & $\begin{array}{l}\text { - falta de lógica e coerência na explicação dos resultados e sua } \\
\text { vinculação aos problemas propostos na pesquisa } \\
\text { - inconsistência teórica na discu ssão dos resultados dos trabalhos de } \\
\text { abordagem qualitativa, em geral a análise é reduzida a explicação } \\
\text { dos depoimentos sem aprofundamento teórico } \\
\text { - incursão em correntes filosóficas sem o devido conhecimento }\end{array}$ \\
\hline Conclusões & $\begin{array}{ll}\text { - } & \text { deslocadas dos objetivos } \\
\text { - } & \text { falta de posicionamento do autor } \\
\text { - } & \text { repetição dos resultados já descritos, não resgata os objetivos } \\
& \text { propostos } \\
\text { - } & \text { deficiência de capacidade reflexiva e perceptiva dos limites do } \\
& \text { estudo }\end{array}$ \\
\hline Resumos & $\begin{array}{l}\text { - } \quad \text { incompletos, desfocados e mal elaborados, não percorrem todas as } \\
\text { etapas e não retratam o estudo realizado } \\
\text { - } \quad \text { resumos apresentando linguagem inadequada e erros de tradução }\end{array}$ \\
\hline Redação & $\begin{array}{l}\text { - } \quad \text { redação confusa e redundante } \\
\text { - } \quad \text { erros de ortografia, gramática e linguagem } \\
\text { - } \quad \text { vocabulário empobrecido } \\
\text { - } \quad \text { falta clareza na expressão das idéias }\end{array}$ \\
\hline $\begin{array}{l}\text { Contribuição para o } \\
\text { desenvolvimento do conhecimento } \\
\text { em Enfermagem }\end{array}$ & $\begin{array}{l}\text { - a maioria das pesquisas apresentadas são descritivas, exploratórias de } \\
\text { contribuição pouco significante quando avaliado o grau de } \\
\text { conhecimento desenvolvido na área } \\
\text { - } \quad \text { falta de elaboração de pesquisas originais em detimento a revisões } \\
\text { - } \quad \text { não oportunizam mudanças de fato na enfermagem } \\
\text { - não contribuem para a produção do conhecimento }\end{array}$ \\
\hline
\end{tabular}

\section{REFERÊNCIAS BIBLIOGRÁFICAS}

INTERNATIONAL COMMITTEE OF MEDICAL EDITORS. Unifor requeriments for manuscripts submitted to biomedical journals. New Eng. J. Med, v.336, p.309-16, 1997. 